\title{
Hydration Water Freezing in Single Supported Lipid Bilayers
}

\author{
Laura Toppozini, ${ }^{1}$ Clare L. Armstrong, ${ }^{1}$ Martin D. Kaye, ${ }^{1}$ Madhusudan Tyagi, ${ }^{2,3}$ \\ Timothy Jenkins, ${ }^{2}$ and Maikel C. Rheinstädter ${ }^{1,4}$ \\ ${ }^{1}$ Department of Physics \& Astronomy, McMaster University, 1280 Main Street West, Hamilton, ON, Canada L8S 4M1 \\ ${ }^{2}$ NIST Center for Neutron Research, NIST, Gaithersburg, MD 20899, USA \\ ${ }^{3}$ Department of Materials Science and Engineering, University of Maryland, College Park, MD 20742, USA \\ ${ }^{4}$ Canadian Neutron Beam Centre, National Research Council Canada, Chalk River, ON, Canada K0J 1 JO \\ Correspondence should be addressed to Maikel C. Rheinstädter, rheinstadter@mcmaster.ca
}

Received 2 January 2012; Accepted 31 January 2012

Academic Editors: J. P. Bradshaw, L. Loura, and J. Perez-Gil

Copyright () 2012 Laura Toppozini et al. This is an open access article distributed under the Creative Commons Attribution License, which permits unrestricted use, distribution, and reproduction in any medium, provided the original work is properly cited.

\begin{abstract}
We present a high-temperature and high-energy resolution neutron scattering investigation of hydration water freezing in single supported lipid bilayers. Single supported lipid bilayers provide a well-defined biological interface to study hydration water dynamics and coupling to membrane degrees of freedom. Nanosecond molecular motions of membrane and hydration water were studied in the temperature range $240 \mathrm{~K}<T<290 \mathrm{~K}$ in slow heating and cooling cycles using coherent and incoherent elastic neutron scattering on a backscattering spectrometer. Several freezing and melting transitions were observed. From the length scale dependence of the elastic scattering, these transitions could be assigned to freezing and melting of hydration water dynamics, diffusive lipid, and lipid acyl-tail dynamics. Coupling was investigated by comparing the different freezing and melting temperatures. While it is often speculated that membrane and hydration water dynamics are strongly coupled, we find that membrane and hydration water dynamics are at least partially decoupled in single bilayers.
\end{abstract}

\section{Introduction}

Despite the vast potential for applications of single membranes in biotechnology and fundamental research $[1,2]$, experiments using single membranes are challenging, mainly because of the small amounts of sample material and corresponding small sample signals. Recent developments in neutron scattering instrumentation, and the increasingly powerful neutron sources, have made it possible to experimentally address molecular dynamics in single bilayers. Lipid diffusion in single supported bilayers made of DMPC (1,2dimyristoyl-sn-glycero-3-phosphatidylcholine) was studied recently using quasielastic neutron scattering [3]. A freezing transition at $T=271 \mathrm{~K}$ was reported and associated with freezing of hydration water molecules. Here, we used solidsupported single-lipid bilayers as a well-defined biological interface to study freezing and melting of hydration water and membrane.
We report a high-energy and temperature-resolution neutron scattering study using a backscattering spectrometer in a temperature range between $240 \mathrm{~K}$ and $290 \mathrm{~K}$. While the temperature range $100 \mathrm{~K}<T<220 \mathrm{~K}$ was investigated thoroughly in the literature to study the so-called "dynamical transition" in hydrated protein and membrane systems, much less attention was paid to the onset of freezing at $271 \mathrm{~K}$ in the past. By analyzing the length scale dependence of the elastic coherent and incoherent scattering, freezing of hydration water and membrane dynamics was observed, and the corresponding freezing and melting temperatures determined. Single supported bilayers of the model membrane system DMPC were prepared on silicon wafers, as shown in Figure 1. Diffusion of lipid molecules, lipid acyl-tail dynamics, and dynamics of hydration water molecules were studied simultaneously using protonated lipids, hydrated by heavy water $\left(\mathrm{D}_{2} \mathrm{O}\right)$. We measured nanosecond molecular dynamics on the High Flux Backscattering Spectrometer (HFBS) at the 


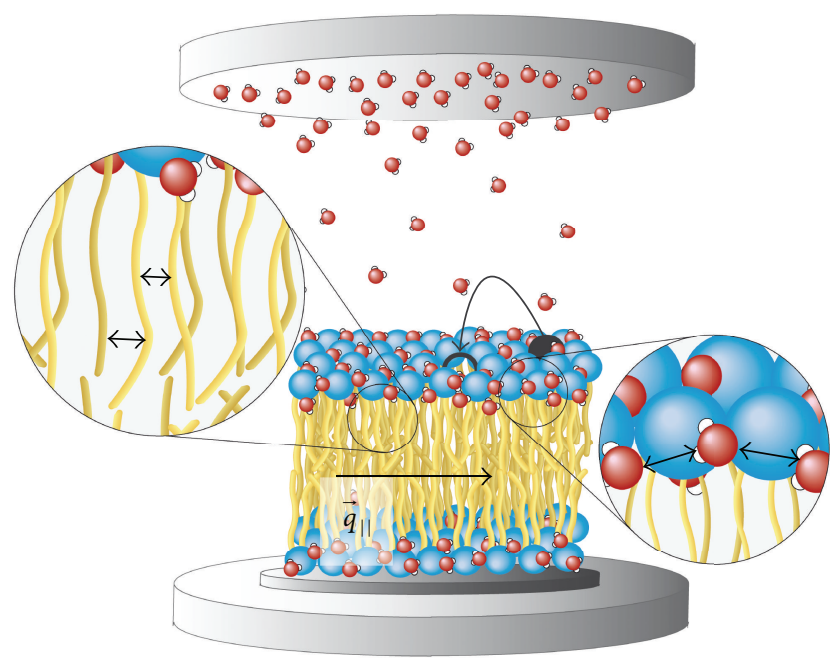

FIGURE 1: Sketch of a hydrated, single supported lipid bilayer, as suggested by [4-6]. Some of the hydration water molecules are drawn. The momentum transfer, $\vec{Q}$, in the neutron scattering experiments was in the plane of the bilayers $\left(\vec{q}_{\|}\right)$to detect in-plane motions of lipid and hydration water molecules. The experiment was sensitive to lipid diffusion, lipid acyl chain and hydration water dynamics.

NIST Center for Neutron Research, Gaithersburg, via elastic neutron scattering experiments. Fixed energy-window scans (FEW-scans) centered at zero energy transfer were recorded as a function of sample temperature to probe molecular motions on a time scale as long as $\sim 1 \mathrm{~ns}$. A freezing transition at $T=271 \mathrm{~K}$ was observed upon slow cooling of the sample. This transition has previously been observed not only in single supported [3] but also in stacked membrane systems [7-10] and was assigned to freezing of membrane hydration water. We find evidence for additional freezing and melting transitions that we assign to hydration water molecules and membrane dynamical degrees of freedom. Coupling between hydration water, lipid diffusion, and lipid acyl-tail dynamics was studied by comparing the corresponding freezing and melting temperatures.

\section{Materials and Methods}

2.1. Sample Preparation. Single supported bilayers of the model system DMPC supported on silicon wafers were prepared by vesicle fusion [11-13] using the protocol reported in [3]: Double-side polished, $2^{\prime \prime} \mathrm{Si}(100)$ - wafers were cleaned by immersing the wafers in an $\mathrm{H}_{2} \mathrm{O}_{2}+$ sulfuric acid mixture (volume fraction of $70 \%$ concentrated $\mathrm{H}_{2} \mathrm{SO}_{4}$, $30 \% \mathrm{H}_{2} \mathrm{O}_{2}$ at $90^{\circ} \mathrm{C}$ for $30 \mathrm{~min}$ ). This strongly oxidizing combination removes all organic contaminants on the surface but does not disturb the native silicon oxide layer. The wafers were then rinsed and stored under distilled water before use [14]. $200 \mathrm{~mL}$ of a $5 \cdot 10^{-3} \mathrm{~mol} / \mathrm{L}$ Hepes, $5 \cdot 10^{-3} \mathrm{~mol} / \mathrm{L} \mathrm{MgCl}_{2}$, and $100 \cdot 10^{-3} \mathrm{~mol} / \mathrm{L} \mathrm{KCl}$ buffer solution was prepared and heated to $55^{\circ} \mathrm{C}$. 1,2-dimyristoylsn-glycero-3-phosphatidylcholine (DMPC) was added to the buffer solution up to a concentration of $1.5 \mathrm{mg} / \mathrm{mL}$. The milky suspension, which initially contained multilamellar lipid suspensions, was sonicated (50\% duty cycle, power level 4) for $15 \mathrm{~h}$ until the suspension became transparent and small unilamellar vesicles formed. The hydrophilic Si wafers were then completely immersed in the suspension for 1 hour. The lipid suspension was kept at $55^{\circ} \mathrm{C}$ during the whole process to keep the bilayers in their fluid phase. After $1 \mathrm{~h}$, the wafers were thoroughly rinsed with $\sim 2 \mathrm{~L}$ of ultra pure water to remove excess vesicles from the surface. By applying this procedure, small bilayer patches initially develop on the substrate, which eventually undergo a transition into a large uniform single bilayer after approximately 20-25 min [12]. The substrates were then annealed for $72 \mathrm{~h}$ at $55^{\circ} \mathrm{C}$ in an oven in air before mounting in an aluminum sample can, and rehydrated. To check the existence of a a single supported bilayer, the quality of the samples was checked by atomic force microscopy (AFM) and X-ray reflectivity. AFM pictures showed a defect-free uniformly covered surface. From the absence of sharp Bragg peaks in the X-ray experiment, a multilamellar structure could be excluded.

To increase the scattering signal, 100 double-sided, polished wafers (resulting in 200 single bilayers) were mounted horizontally into the neutron spectrometer, with the plane of the bilayers in the scattering plane, as depicted in Figure 1. The sample was sealed in an aluminum sample can and placed inside a closed-cycle refrigerator (CCR) during the experiment. The experimental temperature range extended from of $240 \mathrm{~K}$ to $290 \mathrm{~K}$, where freezing of the hydration water molecules was expected. $120 \mu \mathrm{L}$ of heavy water was added before closing to ensure full hydration of the bilayers. The sample can used in this study was designed such that the formation of bulk ice is discouraged in the experimental setup, such as on the windows of the sample can or on the sample, and would occur outside of the neutron beam.

2.2. Neutron Scattering Experiment. The neutron experiment was carried out at the high flux neutron backscattering spectrometer HFBS at the NIST Center for Neutron Research (NCNR), Gaithersburg, [16] in its standard setup with $\mathrm{Si}(111)$ monochromator and analyzer crystals corresponding to an incident and analyzed neutron energy of $2.08 \mathrm{meV}(\lambda=$ $6.27 \AA$ ). At the resolution of $0.8 \mu \mathrm{eV}$, only molecular motions with characteristic times slower than 1 ns are monitored. The experiment covered lateral length scales of $3.5 \AA$ to $12 \AA$ to study in-plane dynamics down to nearest neighbor distances of lipid acyl tails and hydration water molecules. Performing so-called "elastic" scans, which record the elastic scattering intensity as a function of temperature, reveals dynamical changes on different length scales. Freezing and melting of molecular degrees of freedom can easily be identified because they lead to jumps or kinks in the recorded intensity. The counting time per scan was 5 minutes. Using cooling and heating ramps of $0.04 \mathrm{~K} / \mathrm{min}$, this results in a temperature resolution of $0.2 \mathrm{~K}$. The $16 \mathrm{HFBS}$ detectors (D1-D16) cover a total $Q$ range of $0.14 \AA^{-1}$ to $1.81 \AA^{-1}$. The Table 1 lists the center $Q$ values and the corresponding length scales. Each analyzers covers an angular range of approximately $8 \mathrm{deg}$. 
TABLE 1: Neutron detectors (D1-D16), corresponding center $q_{\|}$-value, length scales, and type of scattering and motion detected. The lipid acyl chain correlation peak is centered $q_{\|} \sim 1.5 \AA^{-1}$. The hydration water correlation peak was reported at $q_{\|}=1.85 \AA^{-1}[9,10,15]$.

\begin{tabular}{|c|c|c|c|c|}
\hline Detector & $\mathrm{q}_{\|}\left(\AA^{-1}\right)$ & Lateral distance $(\AA)$ & Dominant scattering & Motion detected \\
\hline D1 & 0.25 & 25 & Coherent small angle scattering & \\
\hline D2 & 0.36 & 17 & & \\
\hline D3 & 0.47 & 13 & & \\
\hline D4 & 0.56 & 11 & & \\
\hline D5 & 0.62 & 10 & & \\
\hline D6 & 0.75 & 8 & & \\
\hline D7 & 0.87 & 7 & Incoherent & Lipid diffusion \\
\hline D8 & 0.99 & 6.3 & & \\
\hline D9 & 1.11 & 5.7 & & \\
\hline D10 & 1.22 & 5.2 & & \\
\hline D11 & 1.32 & 4.8 & & \\
\hline D12 & 1.42 & 4.4 & & \\
\hline D13 & 1.51 & 4.2 & Lipid tail correlation peak & Lipid tail dynamics \\
\hline D14 & 1.60 & 3.9 & & \\
\hline D15 & 1.68 & 3.7 & & \\
\hline D16 & 1.75 & 3.6 & Hydration water correlation peak & Hydration water dynamics \\
\hline
\end{tabular}

As the membranes were oriented with the membrane plane in the scattering plane, the observed $Q$ values were all oriented in the plane of the membranes, $q_{\|}$.

Different types of motion fall into the length scales covered by the HFBS spectrometer. Atomic and molecular motions in membranes can be classified as local, selfcorrelated, and collective, pair-correlated dynamics. Local dynamics correspond to the motion of molecules or functional groups in local energy potentials. The force and time constants involved are determined by the local friction and restoring forces. This type of dynamics is called incoherent because the particles move independently in their local environments. Examples are vibration, rotation, libration (hindered rotation), and diffusion of individual lipid molecules. Coupled, collective molecular motions arise due to an interaction between particles or functional groups. This type of dynamics is called coherent. Collective molecular motions determine, for instance, elasticity of membranes [17] and interactions between membrane embedded proteins [18], and are important for membrane transport $[19,20]$. Within the scattering formalism, incoherent, autocorrelated dynamics is described by the incoherent scattering function, $S_{\text {inc }}(Q, \hbar \omega)$, while the coherent scattering, $S_{\mathrm{coh}}(Q, \hbar \omega)$, describes dynamics involving different molecules $[21,22]$.

Different types of dynamics can be distinguished based on their length scale dependence: Gaspar et al. have recently shown that incoherent and coherent scattering contributions in proteins can be discriminated by using neutron polarization analysis [15]. By using protein powders at different levels of hydration, scattering contributions due to the hydration water could be identified: The nearest neighbor distance of the hydration water molecules leads to a correlation peak centered at $1.85 \AA^{-1}$, corresponding to a distance of
$3.4 \AA$ between hydration water molecules. Detector D16 was, therefore, assigned to dynamics of hydration water molecules.

The lipid acyl chain correlation peak in DMPC is the result of a close packing of the lipid tails in the hydrophobic membrane core. The corresponding correlation peak occurs at $\sim 1.5 \AA^{-1}$ in the gel phase $[10,19]$ (below the main phase transition at $T=294 \mathrm{~K}$ ), corresponding to a nearest neighbor distance of $4.2 \AA$. Detector D13, which covers this length scale, was, therefore, assigned to lipid tail dynamics. Lipid and water dynamics were studied previously using backscattering spectrometers by analyzing elastic scattering at the positions of the lipid acyl chain and hydration water correlation peaks $[9,10,23]$. These studies demonstrated that the different length scales and corresponding detectors are decoupled and that lipid and water dynamics can be studied separately but simultaneously.

Incoherent scattering is dominant at intermediate $q_{\|}$ values, between 0.3 and $1.5 \AA^{-1}$, where no correlation peak occurs. This range of length scales between $\sim 4$ and $21 \AA$ is often used in neutron scattering studies (see, e.g., $[7,8]$ ) to study diffusion and molecular mean square displacements of lipid molecules. We assigned the $Q$ range from $0.6 \AA^{-1}$ to $1.05 \AA^{-1}$ (detectors D5-D8), covering length scales from $6.3 \AA-10 \AA$ to incoherent dynamics due to diffusion of lipid molecules.

Lipid diffusion and lipid and hydration water dynamics were studied simultaneously by analyzing different detectors. Table 1 lists the dominant scattering contribution for the different length scales, and the corresponding type of motion observed in the various detectors. The HFBS analyzers cover an angular range of approximately $8^{\circ}$ each. The experiment was, therefore, not sensitive to shifts of the lipid and water 
correlation peak with temperature within this $q_{\|}$resolution due to potential structural changes of the bilayers with temperature.

\section{Results}

Data were collected during cooling and heating cycles, using a temperature ramp of $0.04 \mathrm{~K} / \mathrm{min}$. The temperature ramp of $0.04 \mathrm{~K} / \mathrm{min}$ was determined to be in thermal equilibrium as there were no observed differences to data taken at even slower speeds. Note that faster ramps could lead to partial dehydration of the membranes because of a possible temperature gradient inside of the sample can.

Figure 2 depicts the elastic scattering collected from detectors D5-D8, D13, and D16, which represent lipid diffusion, (a), lipid tail dynamics, (b), and hydration water dynamics, (c). The elastic scattering due to lipid diffusion increases upon cooling until a freezing transition is observed at $T=264 \mathrm{~K}(3)$. A second step occurs at $T=252 \mathrm{~K}$ (5). The membrane shows a hysteresis in the heating curve, which was shifted upward on the intensity axis for clarity. During the heating cycle, the melting transition is observed to begin at $T$ $=271 \mathrm{~K}(2)$. The membranes transition back into their high temperature state at $279 \mathrm{~K}(1)$.

Lipid tail dynamics were studied in detector D13, which collects scattering at the $q_{\|}$position of the lipid acyl chain correlation peak. The freezing curve in Figure 2(b) shows two pronounced anomalies corresponding to freezing transitions, the first at $T=264 \mathrm{~K}$ (3), and the second at $T$ $=255 \mathrm{~K}(4)$. The temperature of transition (3) in D13 agrees with the transition observed in detectors D5-D8. The heating curve (shifted upward on the intensity axis for clarity) shows transitions at $T=247 \mathrm{~K}(6), T=252 \mathrm{~K}(5), T=271 \mathrm{~K}(2)$ and $T=279 \mathrm{~K}(1)$.

Hydration water dynamics were observed in detector D16 in Figure 2(c). The elastic intensity in Figure 2(c) shows a first kink at $T=271 \mathrm{~K}(2)$ in cooling. A second freezing transition is observed at $T=252 \mathrm{~K}$ (5). Two kinks corresponding to melting transitions can be assigned to the heating curve, at $252 \mathrm{~K}$ and $271 \mathrm{~K}$. We note that the hydration water correlation peak occurs at $q_{\|}=1.85 \AA^{-1}[9,10,15]$. D16 does, therefore, not cover the entire water correlation peak. We argue, however, that D16 covers part of the broad hydration water peak (see [15]) to capture hydration water dynamics. All transition temperatures are listed in Table 2.

An empty aluminum can consisting of silicon wafers (without membranes), and heavy water was measured as a reference. Scattering of the sample, was constant, and no freezing or melting transitions were observed in the temperature range $240 \mathrm{~K}<T<290 \mathrm{~K}$.

The system was cycled several times through the temperature range $240 \mathrm{~K}<T<290 \mathrm{~K}$. Note that the heating curves in Figure 2 have been shifted upward on the intensity axis to distinctly show the transition locations. The original data shows closed loops for all plots, and the system always returned to its initial high-temperature state. The number of particles in the beam was constant, as the intensities of the elastic scattering at the beginning and end of the temperature

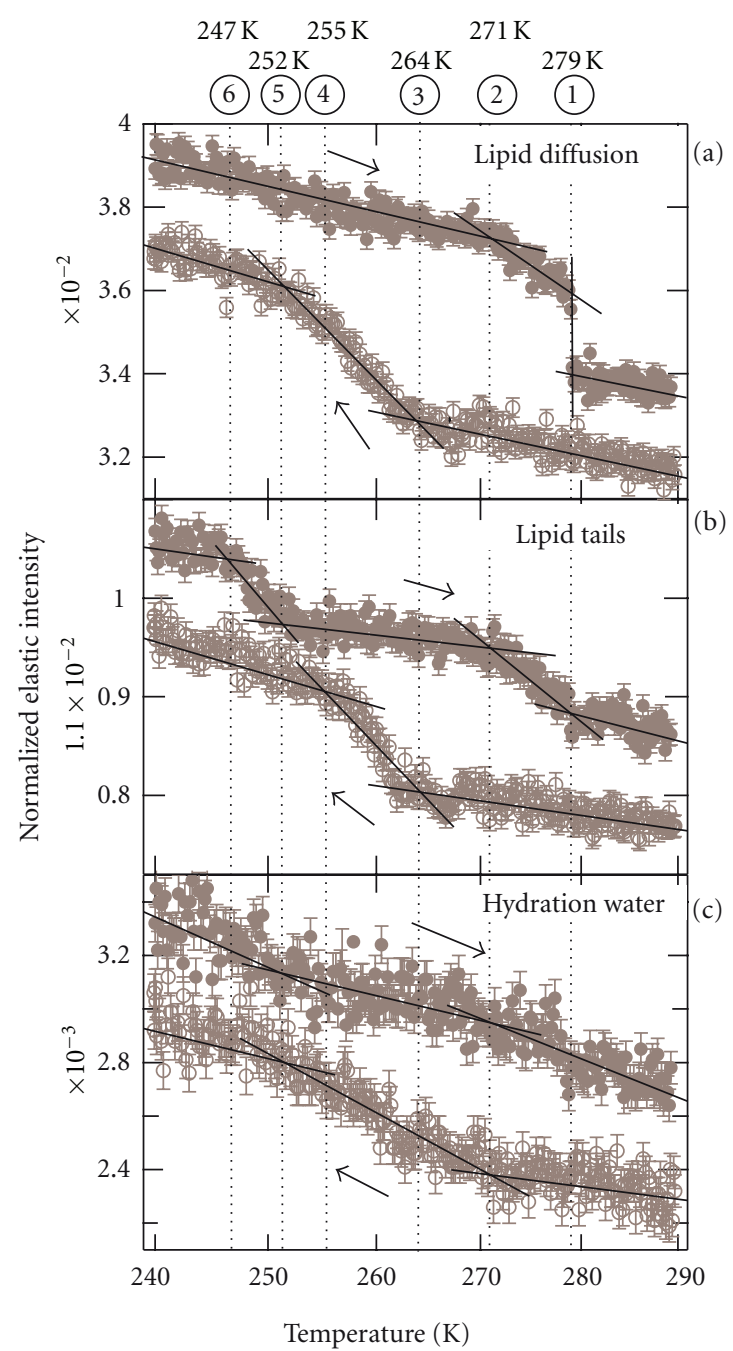

FIGURE 2: Elastic neutron scattering as determined in slow cooling and heating cycles. (a) Detectors D5-D8 cover lipid diffusion. (b) Lipid acyl-tail dynamics were observed in D13. (c) D16 covered hydration water dynamics. Freezing and melting transitions are labeled (1)-(6) and the corresponding temperatures are given. All heating curves are shifted upward in intensity for clarity; the original data show closed loops. Error bars represent one standard deviation. The experimental temperature resolution was $0.2 \mathrm{~K}$. The error in the determination of the transition temperatures is related to the determination of the intersection of the different slopes. For lipid dynamics we estimate the maximum error to be $\sim 2 \mathrm{~K}$. The hydration water data in (c) has slightly larger statistical errors and the maximum error in the determination of the transition temperature was estimated to be $\sim 4 \mathrm{~K}$.

cycle coincide, resulting in perfectly reproducible data. The cooling and heating rate of $0.04 \mathrm{~K} / \mathrm{min}$ was slow enough such that the observed states were thermodynamic states rather than determined by hydration kinetics. The absence of a freezing transition at $T=277 \mathrm{~K}$, the freezing and melting transition of heavy water, in Figures 2(a), 2(b), and 2(c) excludes the presence of bulk $\mathrm{D}_{2} \mathrm{O}$ in the beam. The dynamical changes in Figure 2 were, therefore, attributed to 
TABLE 2: Freezing and melting temperatures of hydration and membrane dynamics in the temperature range $240 \mathrm{~K}<T<290 \mathrm{~K}$ as determined from the data in Figure 2.

\begin{tabular}{lccc}
\hline Motion & Freezing temperature $(\mathrm{K})$ & Melting temperature $(\mathrm{K})$ & Hysteresis $\Delta T(\mathrm{~K})$ \\
\hline \multirow{2}{*}{ Hydration water dynamics } & $271(2)$ & $271(2)$ & 0 \\
& $252(5)$ & $252(5)$ & 0 \\
\hline \multirow{2}{*}{ Diffusive membrane dynamics } & $264(3)$ & $279(1)$ & 15 \\
\hline \multirow{2}{*}{ Membrane dynamics } & $252(5)$ & $271(2)$ & 19 \\
& - & $247(6)$ & - \\
& $264(3)$ & $279(1)$ & 15 \\
\hline
\end{tabular}

dynamical changes of the hydrophobic membrane core and the hydration water molecules.

\section{Discussion}

The onset of freezing at transition (2) in the hydration water scan in Figure 2(c) can be compared to freezing of membrane hydration water in stacked membrane systems as reported from neutron diffraction [7] and elastic neutron scattering experiments [8-10]. The transition temperature of $271 \mathrm{~K}$ agrees well with the temperatures reported. Freezing of these hydration water molecules is supercooled by $6 \mathrm{~K}$ as freezing and melting of bulk $\mathrm{D}_{2} \mathrm{O}$ occurs at $277 \mathrm{~K}$. A second freezing transition was observed at lower temperatures of $T=252 \mathrm{~K}$.

Lipid diffusion and the lipid tail dynamics start to freeze at $T=264 \mathrm{~K}$ (3). The onset of freezing of membrane dynamical degrees of freedom appears to be decoupled from the hydration water freezing. Lipid diffusion shows a second freezing transition at $252 \mathrm{~K}$, which coincides with the lowtemperature hydration water freezing transition. Freezing of the hydration water molecules seems to have a strong effect on the displacement of lipid molecules. Lipid tail dynamics, however, appear to be less sensitive to hydration water dynamics, as the low temperature freezing in Figure 2(b) occurs at $T=255 \mathrm{~K}$, slightly higher than the water freezing. Freezing transitions at $\sim 250 \mathrm{~K}$ were also observed in the elastic neutron scattering and computer simulation data by Wood et al. [8] obtained in purple membrane. However, the relatively coarse temperature resolution in this study does not allow a quantitative comparison with the values in Table 2.

No hysteresis was found in freezing and melting of the hydration water; freezing and melting temperatures coincide in the scan in Figure 2(c). Hysteresis of $15 \mathrm{~K}$ and $19 \mathrm{~K}$ were observed for diffusive membrane and acyl-tail dynamics, as listed in Table 2. These values are significantly larger than the experimental temperature resolution, possible hysteresis of the experimental setup, and the maximum error in the determination of the transition temperatures and must, therefore, be attributed to the membrane.

Diffusive lipid and tail dynamics show a first melting transition at $T=271 \mathrm{~K}(2)$, the temperature of the first hydration water melting transition. Membrane dynamics fully melt and return to their high temperature state at $T=279 \mathrm{~K}(1)$, and diffusive and tail dynamics appear to be coupled in heating. The melting transition of lipid diffusion appears as a pronounced step in the data in Figure 2(a), while all other transitions manifest as kinks. An additional transition at $T=247 \mathrm{~K}$ in the tail dynamics is observed in the heating curve in Figure 2(b), which has no analogue in cooling. Taking into account a possible hysteresis, this points to further freezing transitions in the correlated tail dynamics at lower temperatures, outside the temperature window of this experiment.

As this is the first study of freezing and melting of hydration water and membrane dynamics in solid supported lipid bilayers, we can, at this point, not provide a full explanation and a detailed molecular model. In particular, computer simulations are needed to unambiguously identify hydration water molecules inside the lipid head group region and possibly assign the transitions observed to freezing of water molecules in different hydration shells and their coupling to membrane dynamics. We argue, however, that the two main conclusions of this work are robust: (1) the observation that diffusive and correlated lipid dynamics are to some extent decoupled and show different freezing temperatures, and (2) that hydration water and membrane freezing are at least to some extent de-coupled in single supported lipid bilayers and show different freezing and melting temperatures.

A comment is in order: while one leaflet of the bilayer is directly exposed to water molecules, the other is closely interacting with the silicon substrate, with only a few water molecules available to interact. This is likely to affect the motional freedom of the lipid head groups of the lower layer and their hydration water molecules. The technique presented in this paper is not capable of easily resolving signals from different leaflets. The observed freezing and melting transitions may, therefore, also be related to molecular freezing and melting in different leaflets. It is, for instance, well known that confinement can lead to a suppression or lowering of freezing transitions as a result of competing interactions with the confining surface.

Protein powders, proteins in suspension, or multilamellar membranes are often used as model systems to investigate the structure and dynamics of the hydration water, and 
its impact on membrane and protein dynamics [8, 24-28]. Here, we have for the first time used single phospholipid bilayers supported on a solid surface to study freezing and melting of membrane and hydration water. The upper leaflet of the bilayer, and with it the water adsorption sites in the lipid head group region, is in direct contact with saturated water vapor. By preparing such a well-defined biological interface, it will in future experiments be possible to obtain detailed information about structure and dynamics of the hydration water and its impact on membrane dynamics and dynamics of membrane-embedded proteins.

\section{Conclusion}

In summary, we studied freezing and melting of hydration water and membrane dynamics in single supported lipid bilayers using high energy resolution neutron scattering on a backscattering spectrometer. Membrane and hydration water dynamics were studied in the temperature range $240 \mathrm{~K}<T<290 \mathrm{~K}$ in slow heating and cooling cycles. Several freezing and melting transitions were observed. From the length scale dependence of the elastic scattering, these transitions could be assigned to freezing and melting of the hydration water and freezing of lipid diffusion and lipid acyl-tail dynamics. The different dynamics appear to be partially decoupled in this single bilayer system as we observe different freezing and melting temperatures. The onset of hydration water freezing was found at $T=271 \mathrm{~K}$. Freezing of lipid diffusion and lipid tail dynamics was observed at a slightly lower temperature of $T=264 \mathrm{~K}$. Single-supported lipid bilayers present a well-defined biological interface to study hydration water dynamics and their coupling to membrane degrees of freedom. Because of its simplicity, this system should in particular be highly suited for computer simulations.

\section{Acknowledgments}

The authors thank the NIST Center for Neutron Research for the allocation of beam time. This paper utilized facilities supported in part by the National Science Foundation under Agreement no. DMR-0944772. This paper was partially funded by the Natural Sciences and Engineering Research Council of Canada (NSERC) and the National Research Council Canada (NRC).

\section{References}

[1] E. Sackmann, "Supported membranes: scientific and practical applications," Science, vol. 271, no. 5245, pp. 43-48, 1996.

[2] M. Tanaka and E. Sackmann, "Polymer-supported membranes as models of the cell surface," Nature, vol. 437, no. 7059, pp. 656-663, 2005.

[3] C. L. Armstrong, M. D. Kaye, M. Zamponi et al., "Diffusion in single supported lipid bilayers studied by quasi-elastic neutron scattering," Soft Matter, vol. 6, no. 23, pp. 5864-5867, 2010.

[4] N. Kučerka, Y. Liu, N. Chu, H. I. Petrache, S. TristramNagle, and J. F. Nagle, "Structure of fully hydrated fluid phase DMPC and DLPC lipid bilayers using $\mathrm{x}$-ray scattering from oriented multilamellar arrays and from unilamellar vesicles," Biophysical Journal, vol. 88, no. 4, pp. 2626-2637, 2005.

[5] G. A. Yeghiazaryan, A. H. Poghosyan, and A. A. Shahinyan, "The water molecules orientation around the dipalmitoylphosphatidylcholine head group: a molecular dynamics study," Physica A, vol. 362, no. 1, pp. 197-203, 2006.

[6] E. Nováková, K. Giewekemeyer, and T. Salditt, "Structure of two-component lipid membranes on solid support: an X-ray reflectivity study," Physical Review E, vol. 74, no. 5, Article ID 051911, 2006.

[7] M. Weik, U. Lehnert, and G. Zaccai, "Liquid-like water confined in stacks of biological membranes at $200 \mathrm{~K}$ and its relation to protein dynamics," Biophysical Journal, vol. 89, no. 5, pp. 3639-3646, 2005.

[8] K. Wood, M. Plazanet, F. Gabel et al., "Coupling of protein and hydration-water dynamics in biological membranes," Proceedings of the National Academy of Sciences of the United States of America, vol. 104, no. 46, pp. 18049-18054, 2007.

[9] M. C. Rheinstädter, T. Seydel, F. Demmel, and T. Salditt, "Molecular motions in lipid bilayers studied by the neutron backscattering technique," Physical Review E, vol. 71, no. 6, Article ID 061908, pp. 1-8, 2005.

[10] M. C. Rheinstädter, T. Seydel, and T. Salditt, "Nanosecond molecular relaxations in lipid bilayers studied by high energyresolution neutron scattering and in situ diffraction," Physical Review E, vol. 75, no. 1, Article ID 011907, 2007.

[11] T. H. Watts, A. A. Brian, and J. W. Kappler, "Antigen presentation by supported planar membranes containing affinitypurified I-A(d)," Proceedings of the National Academy of Sciences of the United States of America, vol. 81, no. 23, pp. 7564-7568, 1984.

[12] R. Richter, A. Mukhopadhyay, and A. Brisson, "Pathways of lipid vesicle deposition on solid surfaces: a combined QCM-D and AFM study," Biophysical Journal, vol. 85, no. 5, pp. 30353047, 2003.

[13] V. Oliynyk, U. Kaatze, and T. Heimburg, "Defect formation of lytic peptides in lipid membranes and their influence on the thermodynamic properties of the pore environment," Biochimica et Biophysica Acta, vol. 1768, no. 2, pp. 236-245, 2007.

[14] I. M. Tidswell, B. M. Ocko, P. S. Pershan, S. R. Wasserman, G. M. Whitesides, and J. D. Axe, "X-ray specular reflection studies of silicon coated by organic monolayers (alkylsiloxanes)," Physical Review B, vol. 41, no. 2, pp. 1111-1128, 1990.

[15] A. M. Gaspar, S. Busch, M. S. Appavou et al., "Using polarization analysis to separate the coherent and incoherent scattering from protein samples," Biochimica et Biophysica Acta, vol. 1804, no. 1, pp. 76-82, 2010.

[16] A. Meyer, R. M. Dimeo, P. M. Gehring, and D. A. Neumann, "The high-flux backscattering spectrometer at the NIST Center for Neutron Research," Review of Scientific Instruments, vol. 74, no. 5, pp. 2759-2777, 2003.

[17] M. C. Rheinstädter, W. Häußler, and T. Salditt, "Dispersion relation of lipid membrane shape fluctuations by neutron spin-echo spectrometry," Physical Review Letters, vol. 97, no. 4, Article ID 048103, 2006.

[18] M. C. Rheinstädter, K. Schmalzl, K. Wood, and D. Strauch, "Protein-protein interaction in purple membrane," Physical Review Letters, vol. 103, no. 12, Article ID 128104, 2009.

[19] M. C. Rheinstädter, C. Ollinger, G. Fragneto, F. Demmel, and T. Salditt, "Collective dynamics of lipid membranes studied by inelastic neutron scattering," Physical Review Letters, vol. 93, no. 10, Article ID 108107, 4 pages, 2004.

[20] M. D. Kaye, K. Schmalzl, V. C. Nibali, M. Tarek, and M. C. Rheinstädter, "Ethanol enhances collective dynamics of lipid 
membranes," Physical Review E, vol. 83, Article ID 050907, 4 pages, 2011.

[21] C. L. Armstrong, E. Sandqvist, and M. C. Rheinstädter, "Protein-protein interactions in membranes," Protein and Peptide Letters, vol. 18, no. 4, pp. 344-353, 2011.

[22] M. C. Rheinstädter, "Collective molecular dynamics in proteins and membranes," Biointerphases, vol. 3, no. 2, pp. FB83FB90, 2008.

[23] M. C. Rheinstädter, J. Das, E. J. Flenner, B. Brüning, T. Seydel, and I. Kosztin, "Motional coherence in fluid phospholipid membranes," Physical Review Letters, vol. 101, no. 24, Article ID 248106, 4 pages, 2008.

[24] M. Tarek and D. J. Tobias, "Role of protein-water hydrogen bond dynamics in the protein dynamical transition," Physical Review Letters, vol. 88, no. 13, Article ID 138101, 4 pages, 2002.

[25] D. Russo, G. Hura, and T. Head-Gordon, "Hydration dynamics near a model protein surface," Biophysical Journal, vol. 86, no. 3, pp. 1852-1862, 2004

[26] P. Kumar, Z. Yan, L. Xu et al., "Glass transition in biomolecules and the liquid-liquid critical point of water," Physical Review Letters, vol. 97, no. 17, Article ID 177802, 26 pages, 2006.

[27] S. H. Chen, L. Liu, E. Fratini, P. Baglioni, A. Faraone, and E. Mamontov, "Observation of fragile-to-strong dynamic crossover in protein hydration water," Proceedings of the National Academy of Sciences of the United States of America, vol. 103, no. 24, pp. 9012-9016, 2006.

[28] S. Ebbinghaus, J. K. Seung, M. Heyden et al., "An extended dynamical hydration shell around proteins," Proceedings of the National Academy of Sciences of the United States of America, vol. 104, no. 52, pp. 20749-20752, 2007. 

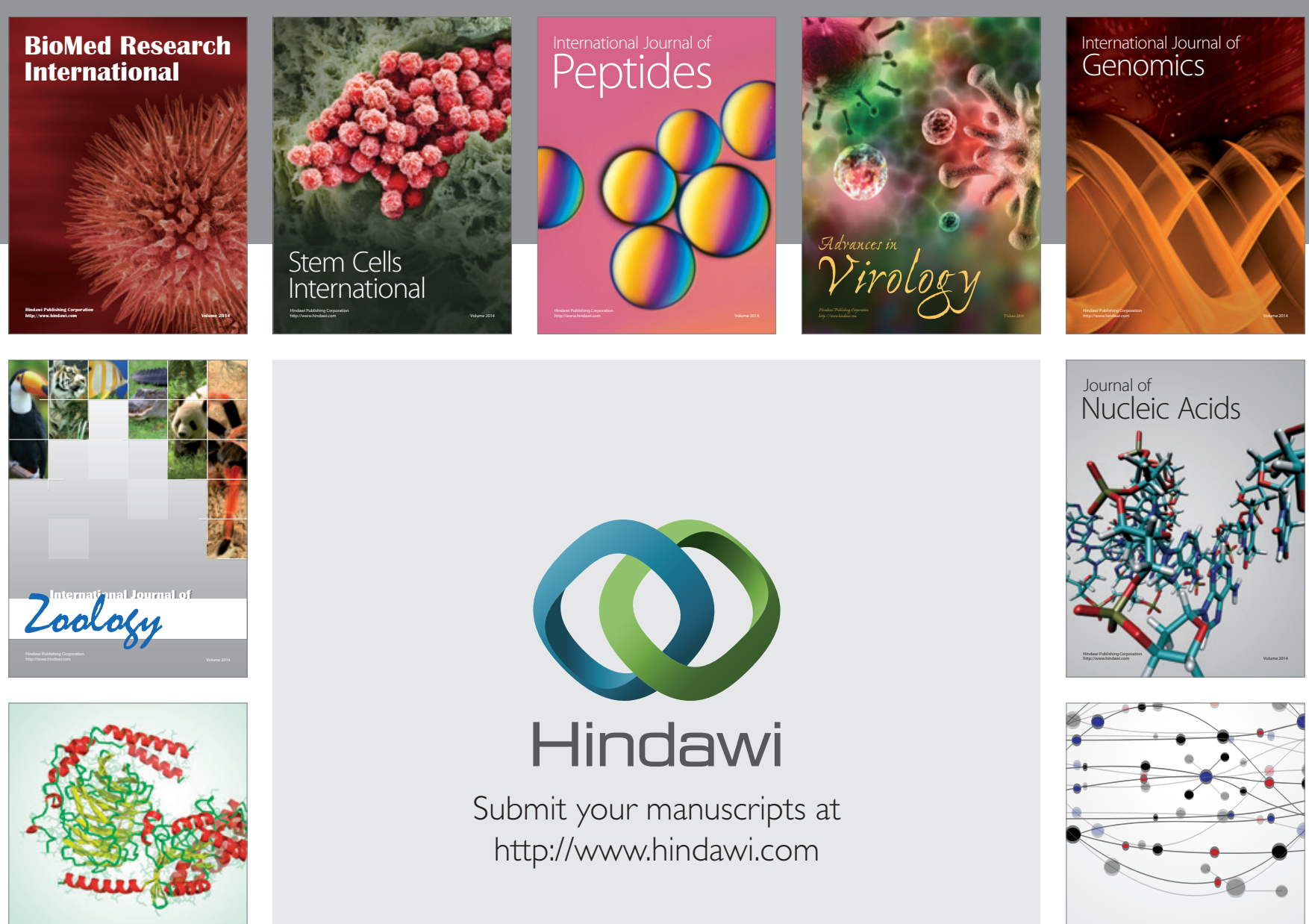

Submit your manuscripts at

http://www.hindawi.com

Signal ${ }^{\text {Jumal }}$ Transduction
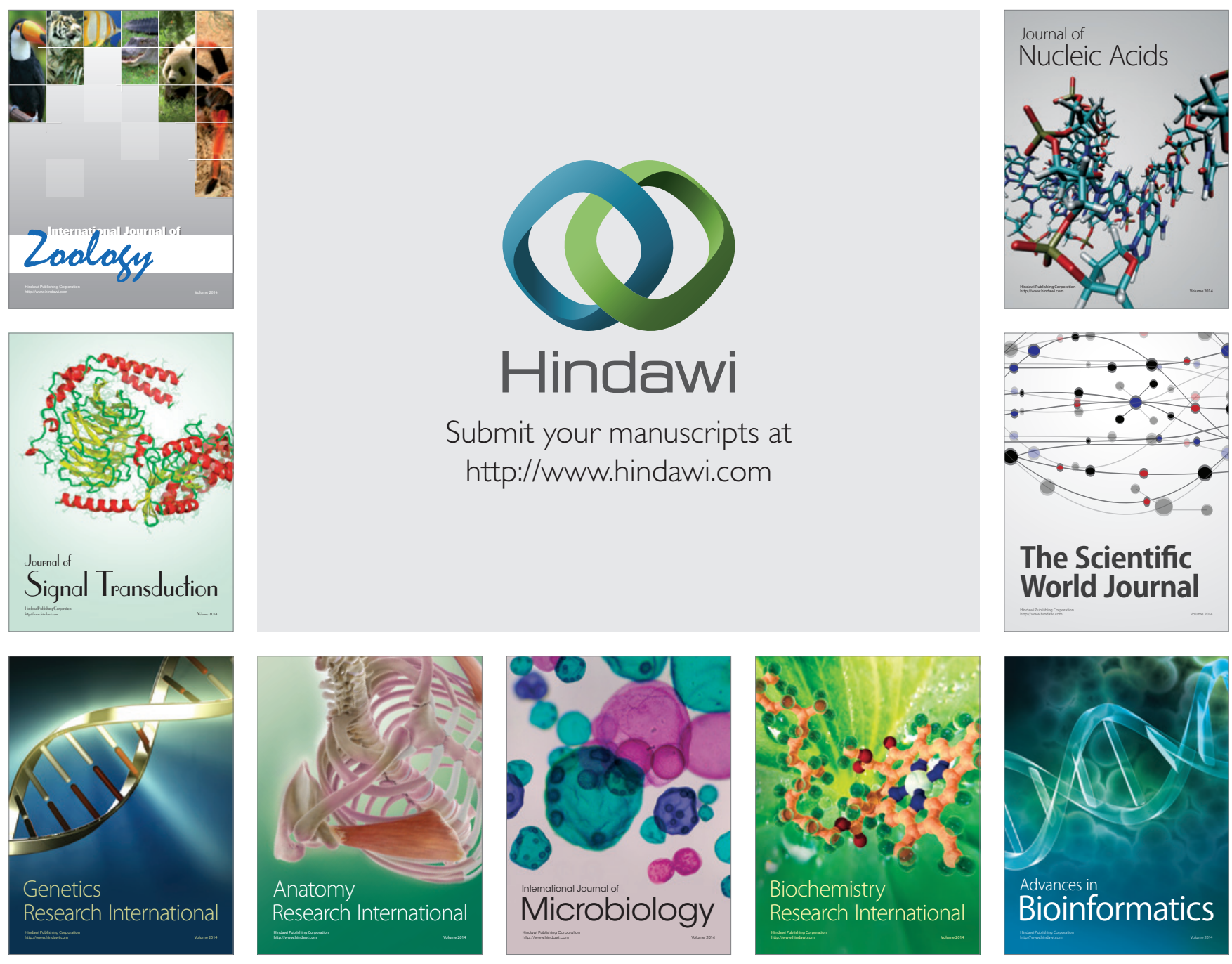

The Scientific World Journal
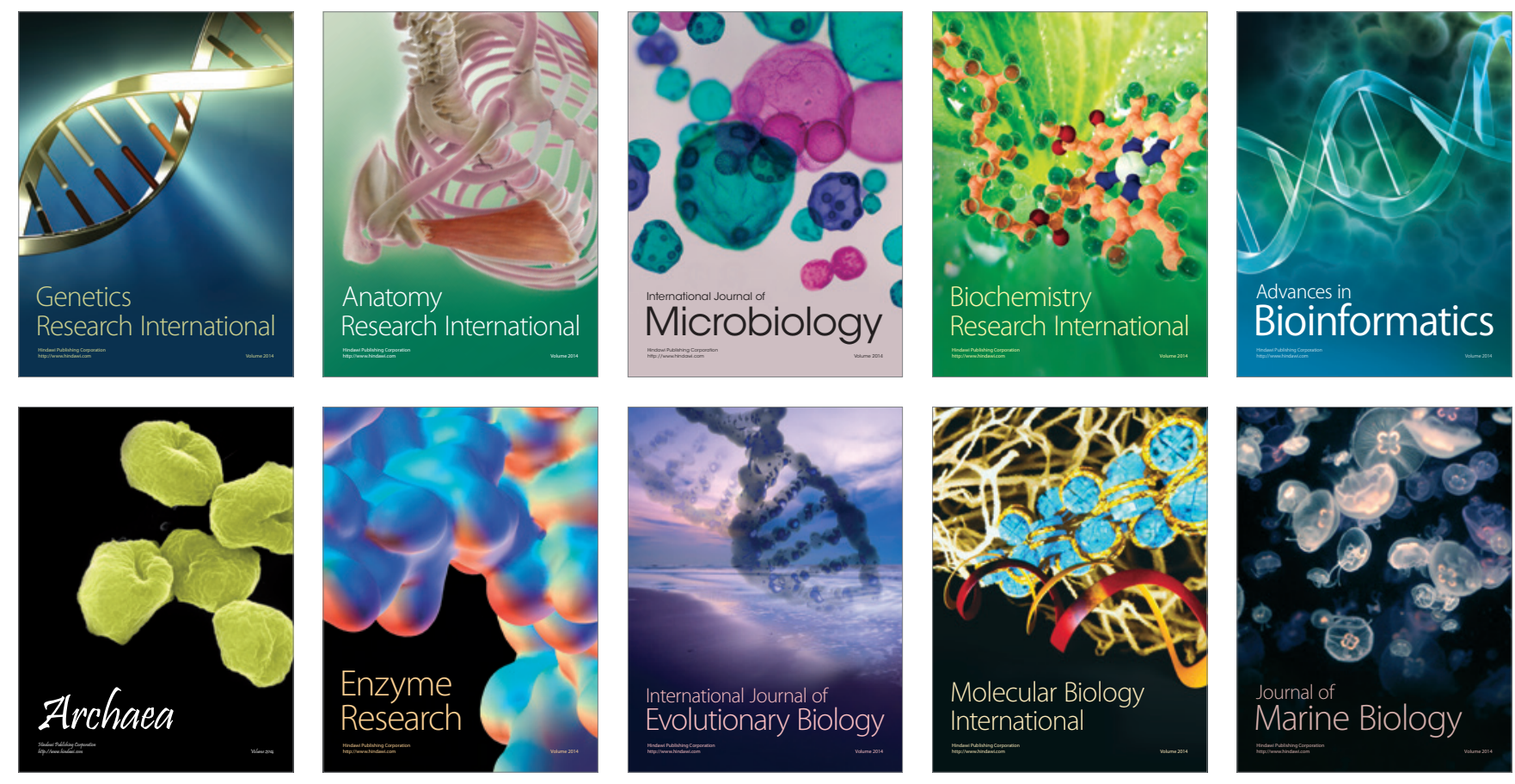